Regionen zwischen Kooperation und Konkurrenz

\title{
Zwei Seiten einer Medaille
}

\section{Regionale Kooperation hat aus nahe liegenden Gründen Konjunktur. Insbesonde- re komplexe regionale Nachhaltigkeitsstrategien lassen sich nur so durchsetzen. Gleichzeitig nimmt aber auch die Konkurrenz zwischen den Regionen zu. Dies ist gut so, da erst hierdurch systematische Kooperationsgewinne ermöglicht wer- den. Erst ein Zusammenspiel beider Steuerungsprinzipien kann ihre jeweiligen Grenzen überwinden (1).}

$\mathrm{R}$ Von Eckhard Bergmann egionale Kooperation hat Konjunktur. Sie wird von vielen Akteuren in vielen Regionen gewollt, Ausnahmen sind eher selten. Sie wird von der bundesdeutschen Raumordnung unterstïtzt, etwa durch die Förderung von Regionalkonferenzen oder des „Netzwerkes Regionen der Zukunft“. Die Gründe sind naheliegend:

Auf der regionalen Ebene „können die Folgen des eigenen Handelns unmittelbarer erfahren und verantwortet werden, hier lassen sich viele ökologische und ökonomische Zusammenhänge überschaubar gestalten, hier lassen sich dezentral in vernetzten Strukturen viele Probleme effizienter lösen, hier könnte eine breite öffentliche Diskussion über die gemeinsame Zukunft gelingen“(2). Insbesondere komplexe Regionalentwicklungsstrategien - Stichwort Nachhaltigkeit - lassen sich (nur) so durchsetzen. Regionale Kooperationen schaffen einen Spielraum für räumlich angepasste Lösungen, bei denen das Land tendenziell überfordert wäre und zu denen die Gemeinden aus Eigeninteresse vielfach nicht bereit sind. Eine derartige Kooperation ist nichts anderes als die Suche nach Tauschgewinnen und immer dann, wenn solche Gewinne erzielt werden können, ist die Vermutung nahe liegend, dass dies auch mit Vorteilen für die Gesellschaft insgesamt verbunden ist.

Gleichzeitig nimmt aber auch die Konkurrenz zwischen den Regionen zu, die Erweiterung der EU wird diesen Prozess weiter verstärken. Und dies ist gut so: Regionaler Wettbewerb ist ein Teil des föderalen Wettbewerbs und regt so zu einem optimalen Einsatz der in einer Gebietskörperschaft vorhandenen Ressourcen an, indem er Kostensenkungen und Qualitätsverbesserungen hervorruft. Dort wo der Wettbewerb funktioniert - und dies ist eine bedeutsame Einschränkung - fördert er gesellschaftliche Zusammenarbeit zum gegenseitigen Vorteil.
Dies gilt auch in dynamischer Perspektive: Entdeckungsverfahren für Produkt- und Prozess-Innovationen werden angeregt. Die regionalen Akteure werden angehalten, ihre Konzepte, Organisationsformen und Maßnahmen weiter zu entwickeln; ein Verweilen auf dem Erreichten schwächt perspektivisch die Konkurrenzfähigkeit.

\section{Zusammenspiel beider Steuerungsprinzipien}

Kooperation und Wettbewerb weisen jeweils unterschiedliche Vorzüge auf. Sie verhalten sich aber nicht zueinander wie Feuer und Wasser, sondern sie sind letztlich zwei Seiten einer Medaille (3). Bei der Kooperation wird eine Erweiterung der Handlungsmöglichkeiten angestrebt, um im Wettbewerb der Regionen bestehen zu können - die Konkurrenz zwischen Gemeinden bzw. Regionen ist letztlich das entscheidende Argument für eine regionale Kooperation.

Jedes Steuerungsprinzip - egal ob Kooperation oder Wettbewerb - muss dabei die Interessen der Akteure respektieren. Dabei ist davon auszugehen, dass die Knappheit der Ressourcen zu Interessenkonflikten führt. Quasi im Gegenzug resultieren aber aus der Möglichkeit, über die Verfolgung gemeinsamer Interessen Vorteile zu erzielen, die bekannten Formen der sozialen Interaktion wie Tausch oder Kooperation.

Auch der Wettbewerb zwischen Gebietskörperschaften um BürgerInnen und Unternehmen is ein Wettbewerb um Kooperationschancen. „Produktiv wird dieser Wettbewerb, wenn die Konkurrenten über Leistungen konkurrieren, die der anderen Marktseite Nutzen stiften. Die andere Seite wirkt dabei als Selektionsinstanz, und dieser Umstand zwingt die Konkurrenten, ihre Leistungen an deren Interessen auszurichten."(4)

Dies kann - insbesondere kleinere - Gebietskörperschaften zwingen, Kooperationen einzugehen, um den Anforderungen des institutionellen Wett- bewerbs gerecht werden zu können. Konkurrenz und Kooperation werden so mächtige Verbündete: Wenn man den Konkurrenten nicht besiegen kann, dann muss man sich verbünden. „Coopetition" lautet nun das Zauberwort.

\section{Grenzen der Kooperation}

Aber auch wenn man sich für ein Zusammenspiel von regionaler Kooperation und regionalem Wettbewerb ausspricht, so darf man doch nicht die Grenzen vergessen, die beiden Steuerungsprinzipien zu eigen sind. Kooperationen werden etwa nur dann eingegangen, wenn die Teilnehmer sich einen Nutzen aus dieser Kooperation versprechen. Dies ist relativ einfach bei Win-Win-Situationen. Sobald Verteilungskonflikte vorliegen, werden Kooperationen seltener. Aber, und hier wird es spannend, existierende, gewachsene Kooperationsroutinen aus Win-Win-Feldern können dazu führen, auch in jenen Feldern zu kooperieren, in denen die Verteilungsfolgen problematischer sind.

Dies gilt insbesondere bei regionalen öffentlichen Gütern. Wenn beispielsweise die potenziellen Kooperationspartner auch ohne aktiven Beitrag in den Genuss des kooperativ erstellten Gutes kommen, sinkt ihre Kooperationsbereitschaft. Wenn keiner von der Nutzung ausgeschlossen werden kann, warum sollte man sich dann an der (Opportunitäts-)Kosten verursachenden Kooperation beteiligen? Andererseits wissen die Nutzer, dass ohne die Finanzierungsbeiträge der Free-Rider das - auch von ihm als wünschenswert angesehene - öffentliche Gut nicht bereit gestellt wird. Dass sich am Ende alle schlechter stellen, ist das bekannte Ergebnis des Gefangenendilemmas. Dies erklärt auch, warum beispielsweise Kooperationen im Management der Freiflächen noch spärlich sind, denn hier sind positive externe Effekte ausgeprägt. Warum soll sich die Gemeinde an solchen Aufgaben beteiligen, wenn die Freiflächenfunktionen - sind sie erst einmal bereitgestellt - umsonst genutzt werden können? Gleiches gilt für die Probleme, die aus der nach wie vor anhaltenden Suburbanisierung entstehen. Die Abwanderung von Einwohnern und Unternehmen führt zu sinkenden Steuereinnahmen bei gleichzeitig wachsenden finanziellen Lasten in den Kernstädten. Hier einen angemessenen Ausgleich über Kooperation zu erreichen, erscheint allerdings außerordentlich schwierig, profitiert das Umland doch vermeintlich auch ohne eigenes Zutun von den städtischen Leistungen.

Darüber hinaus müssen die Gewinne aus der Kooperation hoch genug ausfallen, um die entstehen- 
den Koordinationskosten zwischen den Kooperationspartnern zu kompensieren. Diese Transaktionskosten, also Verhandlungs-, Informations- und Durchsetzungskosten, können aber leicht prohibitiv hoch werden. Hinzu kommt eine zeitliche Asymmetrie: Kooperationen sind auf der einen Seite aktuell mit Kosten und Risiken verknüpft, politischer Einfluss und finanzielle Ressourcen werden abgetreten. Dem stehen - teilweise erst mittelfristig - nicht leicht kalkulierbare Nutzen gegenüber.

Kooperationen können aber noch aus einem weiteren Grund problematisch sein. Sie werden dann ordnungspolitisch bedenklich, wenn sie als Bündnisse zu Lasten Dritter eingegangen werden. In dem Maße, in dem sie Wettbewerb verhindern, reduzieren sie auch den Wettbewerbsdruck und damit die Anreize für die politischen Akteure zu effizientem und innovativen Verhalten. Kooperation, die nicht in Wettbewerb eingebettet ist, läuft leicht Gefahr, zu einem regionalen Kartell zu werden. Auch aus diesem Grund bedarf das Steuerungsprinzip Kooperation der Ergänzung durch das Steuerungsprinzip Wettbewerb.

\section{- Grenzen des Wettbewerbs}

Aber auch das Steuerungsprinzip Wettbewerb hat Grenzen. Denn föderaler Wettbewerb lässt sich zum einen nicht umstandslos mit dem marktlichen vergleichen: Unternehmen finanzieren sich über Verkaufserlöse und Fremdkapital und tragen das volle (Insolvenz-) Risiko; politische Akteure dagegen arbeiten ,mit Hilfe fremder Ressourcen“ (5) und tragen maximal das Abwahl-Risiko. Dies birgt die Gefahr, dass - aus gesamtwirtschaftlicher Sicht - zu viele Mittel in die Akquisition von privaten Investoren und Bürgern gesteckt werden. $\mathrm{Zu}$ bedenken ist, dass jeder für einen aktiven Wettbewerb eingesetzte Euro damit der nächstbesten Verwendung entzogen wird.

Zum anderen - wieder anders als beim Markt gibt es die Befürchtung, dass sich eine ruinöse Konkurrenz entwickeln kann. Wie wahrscheinlich ein derartiges Race-to-the-Bottom ist, ist umstritten. Befürchtet wird, dass die politischen Akteure sich dem ökonomischen Standort-Imperativ beugen müssen. Der institutionelle Wettbewerb endet dann letztlich in einem Negativ-Wettlauf zwischen den Regionen: Wenn die Regionen um mobiles Kapital, mobile Unternehmen und mobile Arbeitnehmer konkurrieren, werden zu geringe Steuersätze, zu hohe Subventionen, zu geringe Umweltstandards usw. festgelegt. Dass dies insbesondere für eine nachhaltige Regionalentwicklung zum Problem werden kann, liegt auf der Hand.

\section{Fazit}

Trotz aller dieser Kritik spricht dennoch einiges dafür, dass per Saldo ein gestärkter - nicht uneingeschränkter - föderaler Wettbewerb gute Steuerungsleistungen erbringt, weil er eine disziplinierende Funktion ausübt und - bei gut definierten Spielregeln - ein effizientes Instrument auf der Suche nach richtigen Lösungen ist. Ähnliches gilt für die regionale Kooperation. Trotz kritischer Einwände ist sie in den Handlungsfeldern, in denen sie ihre Leistungsfähigkeit ausspielen kann, ein unabdingbarer Bestandteil einer nachhaltigen Regionalentwicklung.

Für beide Steuerungsprinzipien gilt, wie für alle Instrumente, dass man sich selbst keinen Gefallen tut, wenn man die daraus erwachsenden Vorteile zu gering erachtet oder wenn man die sich ergebenden Handlungsspielräume überschätzt. Befürworter und Gegner - immer noch sind die Befürworter kooperativer Prozesse oft große Marktskeptiker und vice versa - greifen zu kurz, wenn sie ihre jeweilige Steuerungs-Perspektive verabsolutieren. Nur wenn die in den jeweiligen Steuerungsprinzipien liegenden Potenziale mobilisiert, gleichzeitig aber auch ihre Grenzen beachtet und respektiert werden, kann Regionalentwicklung als dezentrale und ökologische Strategie erfolgreich sein.

\section{Anmerkungen}

(1) Der Autor gibt in diesem Beitrag seine persönliche Meinung wieder.

(2) So schon klassisch Schleicher-Tappeser, R. u.a.: Perspektiven ökologischer Regionalentwicklung und Südbaden. Pfaffenweiler 1992, S. 1.

(3) Siehe hierzu und zum folgenden Bergmann, E./ Jakubowski, P.: Strategien der Raumordnung zwischen Kooperation und Wettbewerb. In: Informationen zur Raumentwicklung, Heft 8/2001.

(4) Suchanek, A.: Nachhaltigkeit und Wettbewerb. In: Renner, A./ Hinterberger, F. (Hrsg.): Zukunftsfähigkeit und Neoliberalismus. Baden-Baden 1998, S. 204.

(5) Benkert, W.: Interkommunale Konkurrenz. Formen, Ergebnisse und Bewertung von Wettbewerb im Staatssektor. In: Postlep, R.-D. (Hrsg.): Aktuelle Fragen zum Föderalismus. Marburg 1996, S. 173.

\section{Der Autor}

Eckhard Bergmann ist Leiter des Referats 14 Wirtschaft und Gesellschaft des Bundesamts für Bauwesen und Raumordnung (BBR).

Kontakt: BBR, Deichmanns Aue 31-37, 53179 Bonn. Tel. 01888-401-2340, Fax -2346,

E-mail: Eckhard.Bergmann@bbr.bund.de

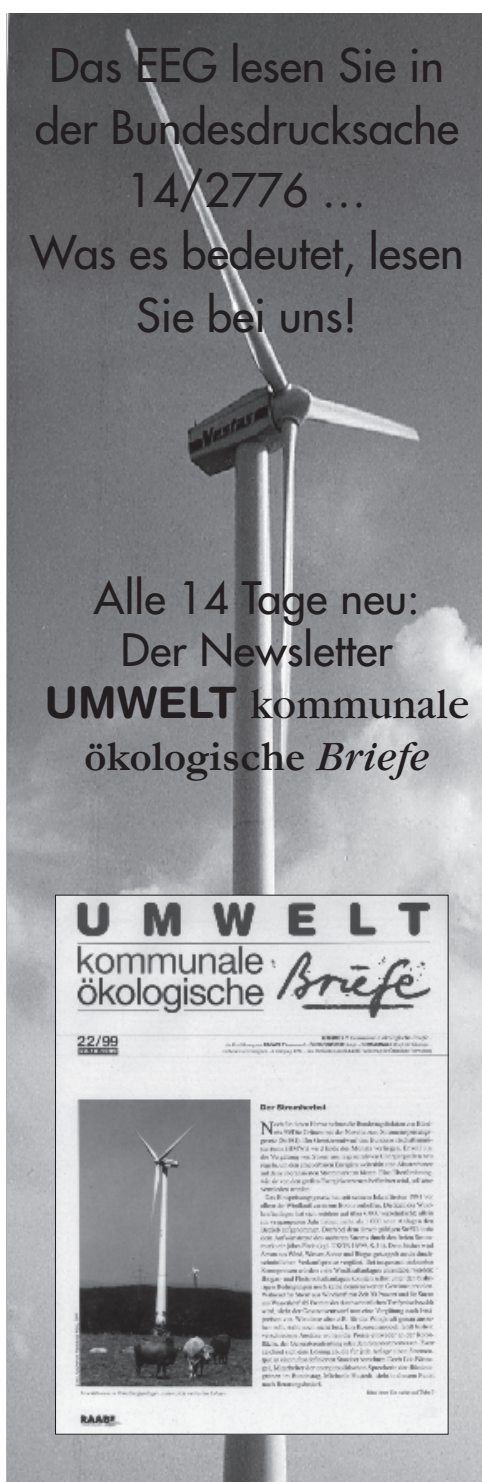

Fordern Sie gleich ein kostenloses Probeabo an!

Schicken Sie den Coupon bitte an:

UmweltBriefe, Raabe Fachverlag Kaiser-Friedrich-Str. 90, D-10585 Berlin Fon 030/212987-23 Fax 030/21 2987-20 E-mail UMWELTBRIEFE@raabe.de Internet http://www.umweltbriefe.de

Ja, senden Sie mir die nächsten drei Ausgaben von UMWELT kommunale ökologische Briefe kostenlos zu. Wenn Sie eine Woche nach Erhalt des letzten Freiexemplares nichts von mir hören, bestelle ich bis auf weiteres, mindestens aber für ein Jahr, UMWELT kommunale ökologische Briefe. Der Jahresbezugspreis für ein Abonnement (26 Ausgaben) beträgt $360 \mathrm{DM}$ (unverb. Preisempf.) zzgl. Versandspesen.

Name:

Straße:

PLZ, Ort:

Datum. Unterschriff*):

Ich bin/wir sind berechtigt, die Bestellung des Abonnements ohne Angaben von Gründen innerhalb einer Woche nach Erhalt der drei Freiexemplare in schritlicher Form zu wider den. Zur Widents (Poststell.

Datum: Unterschriff*)

*) Bitte an beiden Stellen unterschreiben 
(c) 20I0 Authors; licensee IÖW and oekom verlag. This is an article distributed under the terms of the Creative Commons Attribution Non-Commercial No Derivates License (http://creativecommons.org/licenses/by-nc-nd/3.o/), which permits unrestricted use, distribution, and reproduction in any medium, provided the original work is properly cited. 